\title{
Modularizando y desmodularizando la cognición social: el caso de la detección de trampa
}

\section{Modularizing and De-modularizing Social Cognition: The Cheating Detection Module Case}

\author{
PAOLA HERNÁNDEZ-CHÁVEZ, JONATAN GARCÍA-CAMPOS \\ UNIVERSIDAD DE JUÁREZ DEL ESTADO DE DURANGO
}

Recibido: 23/12/2019 Aceptado:12/07/2020

\begin{abstract}
RESUMEN
En este trabajo, revisamos la literatura clásica en modularidad cognitiva. Ofrecemos una descripción cognitiva neuronal de la modularidad, que incluye: patrones de disfunción específicos, la doble disociación, una actividad cerebral asociada, y un proceso de especialización funcional. Posteriormente, analizamos un módulo de cognición social, i.e., el módulo de detección de tramposos. Compilamos las objeciones clásicas a este módulo, y ofrecemos algunas nuevas, para posteriormente contrastarlas con las primeras. En la última parte, ofrecemos algunas implicaciones y direcciones futuras para los estudios actuales sobre cognición social.
\end{abstract}

\author{
PALABRAS CLAVE \\ COGNICIÓN SOCIAL, MODULARIDAD, MODULARIDAD MASIVA, \\ ESPECIALIZACIÓN CEREBRAL, DETECCIÓN DE TRAMPA
}

\begin{abstract}
This work reviews the classic literature on cognitive modularity. A neuronal cognitive account of modularity is offered, which includes: specific breakdown patterns, double dissociation, an associated brain activity, and a process of functional specialization. Thereupon, a social cognition module is analyzed, i.e., the cheating detection module. The classic objections to
\end{abstract}

(C) Contrastes. Revista Internacional de Filosofía, vol. XXV No2 (2020), pp. 57-78. ISSN: 1136-4076

Departamento de Filosofía, Universidad de Málaga, Facultad de Filosofía y Letras Campus de Teatinos, E-29071 Málaga (España) 
this module are compiled, while some original ones are displayed, and then contrasted with the former account. The last part advances some implications and future directions for current social cognition studies.

\author{
KEYWORDS \\ SOCIAL COGNITION, MODULARITY, MASSIVE MODULARITY, BRAIN \\ SPECIALIZATION, CHEATING DETECTION
}

\title{
I. LA MODULARIDAD COGNITIVO-NEURONAL
}

LA NEUROCIENCIA SOCIAL ES la búsqueda por comprender los mecanismos biológicos que subyacen a la conducta social. El análisis que presentamos se suscribe a tal búsqueda en tanto que analiza críticamente la fuerza y pertinencia de la evidencia neurocientífica utilizada por los psicólogos evolucionistas para sostener que los humanos poseemos un mecanismo modular que nos permite detectar quién viola un contrato social. Ofreceremos una noción cognitivoneuronal de la modularidad que pone en duda, por buenas razones, la existencia de módulos como el de detección de tramposos.

\section{I.1. MODULARIDAD DE LA MENTE PERIFÉRICA Y MASIVA}

Uno de los temas más importantes en la filosofía de las ciencias cognitivas es la pregunta: ¿cuál es la estructura de la mente? Una respuesta a esta pregunta fue ofrecida por una familia de teorías conocidas como las arquitecturas modulares de la cognición. La discusión analítico-filosófica respecto a qué es un módulo fue planteada por Jerry Fodor (1983). Él sostuvo que sólo tenemos módulos o sistemas de insumo en la periferia de la mente, y que éstos constituyen partes esenciales de los sentidos y el lenguaje. Antes de los módulos, están los cables físicos que los alimentan, a saber, los transductores. Estos captan las impresiones sensoriales directas, son partes físicas, por ejemplo, los tímpanos y los bastones retinales. El resto de la mente, dicho filósofo lo denomina «procesador central», pues no se compone de módulos sino de mecanismos generales que cruzan dominios, comparten recursos e intercambian información, recombinándola para producir cosas tan complejas como los pensamientos y la fijación de creencias.

Entonces, los mecanismos de entrada son los cables físicos o transductores que alimentan a los siguientes componentes, a saber, los módulos, que a su vez alimentan al procesador central, donde se dan los productos complejos de la mente. A lo que Fodor (1983) dedicó mayor atención fue a la descripción de los módulos, los cuales tienen las siguientes características, son: específicos de dominio, involuntarios, tienen acceso limitado a información de otros 
procesadores, son rápidos, están encapsulados informacionalmente, emiten respuestas superficiales, están asociados a una arquitectura neural, exhiben patrones típicos de disfunción y tienen una ontogenia característica.

Como bien señaló Fodor (1983), y como sigue siendo cierto para defensores y detractores, la modularidad es una cuestión de grados. Es decir, la modularidad depende de en qué medida se satisface o no las características que permiten considerar al mecanismo como un módulo. De modo que entre más características satisfaga el mecanismo cognitivo, más modular se le considerará.

Frente a la propuesta fodoriana se presentaron propuestas alternativas como la modularidad masiva. Los modularistas masivos -entre los cuales hay diversos filósofos (Sperber 1994, Carruthers 2006, Machery 2007), psicólogos (Cosmides y Tooby, 1996), lingüistas (Pinker 1994) y otros científicos cognitivos (Mithen, 1996)- consideran que toda o la mayor parte de la mente está compuesta por módulos. Estos estudiosos de la cognición no concuerdan con los rasgos que Fodor había formulado para los módulos, lo que en parte explica el debate acerca de qué tan modular es la mente. Un rasgo fundamental de la noción de módulo según los modularistas masivos es que éstos son producto de la selección natural (Cosmides \& Tooby 1992, Sperber 1994, Carruthers 2006, Machery 2007) y que seguramente el poseerlos debió representar alguna ventaja adaptativa.

Es así como la literatura en torno a la arquitectura de la mente comenzó por dividirse entre aquellos que consideraban que sólo una pequeña parte de la mente eran modular, y aquellos que pensaban que toda o casi toda la mente lo es.

En esta trabajo, no nos centraremos en el debate entre la propuesta fodoriana y la modularista masiva, más bien deseamos presentar y defender una noción de módulo articulada y mas plausible que siga siendo de utilidad para la neurociencia social y las ciencias cognitivas en general. En el próximo apartado, presentamos un enfoque de la modularidad cognitiva que es tanto teórica como empíricamente viable.

\section{I.2. UNA NOCIÓN EMPÍRICA DE MÓDULO}

En esta sección, explicamos cada uno de los rasgos de una noción de módulo cognitivo-neuronal empíricamente viable. Con esta noción, sostenemos que un mecanismo cognitivo es mas probablemente modular entre mas satisfaga las siguientes características: (1) la presencia de patrones típicos de disfunción, (2) es producto de disociaciones dobles, (3) posee evidencia de actividad cerebral específica, $y,(4)$ puede entenderse como un proceso de especialización cerebral y cognitiva. 


\section{I.2.1. LAS DISFUNCIONES}

Un elemento neurocientífico importante dentro de la modularidad cognitiva es la presencia de disfunciones o fallas neurológicas selectivas. Esta característica de la modularidad se encontraba presente desde la formulación de Fodor (1983), cuando afirmaba que los módulos cognitivos son propensos a síndromes de disfunción bien definidos, a partir de lo cual suponía que las patologías de los sistemas modulares son causadas por averías en los circuitos cerebrales especializados (Fodor, 1983, pp. 99-100). Este rasgo ha tomado un rol central en años recientes.

La presencia de lesiones cerebrales particulares se correlaciona con fallas neuropsicológicas específicas. De hecho, evidencia importante a favor de que la arquitectura cognitiva modular proviene, precisamente, de pacientes con daño cerebral focalizado. El móvil de este razonamiento es que, si un daño cerebral focalizado afecta sólo a una función cognitiva sin afectar al resto de las funciones, seguramente es porque la función dañada depende o se correlaciona directamente esa estructura cerebral dañada. Esta característica hace plausible suponer que las funciones cognitivas están segregadas y diferenciadas.

\section{I.2.2. LAS DISOCIACIONES DOBLES}

Formulado de manera simple, la disociación es un método que se utiliza en psicología y neuropsicología, que se basa en el estudio de pacientes con déficits cognitivos, ya sean aquellos que son resultado de un problema del neurodesarrollo o de una lesión cerebral. Una disociación simple ocurre cuando, tras sufrir un daño en una región cerebral, llamémosla $R x$, se observa que un grupo de pacientes, digamos $S 1$, presenta un deterioro cognitivo durante el desempeño de la tarea $t A$, pero no presenta fallas (considerables) al desempeñar la tarea $t B$ o las demás tareas. Con esto se puede inferir que la $t A$ se encuentra dañada selectivamente y disociada del resto de las demás tareas.

Puesto que una disociación simple es insuficiente como método de prueba, se necesita un recurso confirmatorio o cruzado para fundamentar la independencia funcional de las áreas cerebrales asociadas al desempeño de una tarea cognitiva. Una disociación doble, o cruzada, ocurre cuando, además de que ocurre una disociación simple, tenemos otro grupo de pacientes $S 2$ que presentan un daño cerebral en la región $R y$ y un deterioro cognitivo durante el desempeño de la tarea $t B$, pero no presenta fallas (considerables) al ejecutar la tarea $t A$. A partir del cruzamiento de disociaciones en ambos grupos, se infiere que la $t A$ y la $t B$ están separadas.

Como ejemplo, supongamos que tenemos a un grupo de pacientes $A$ con una lesión en el área de Broca; y que tenemos también a un grupo de pacientes $B$ con una lesión en el área de Wernicke. Después de pedirles que desempeñen 
una tarea con el fin de evaluar la comprensión del habla y la emisión de palabras habladas, se tiene que: el grupo $A$, con lesión en el área de Broca, no es capaz de hablar o lo hace con mucha dificultad, pero sí entienden el habla de otros (aquellos que padecen afasia no fluida o afasia de Broca-motora); mientras el grupo $B$, con lesión en el área de Wernicke, presentan habla fluida, muchas veces desprovista de sentido, pero no tienen comprensión de la misma (afasia fluida o de Wernicke-sensorial). A partir de esto se puede inferir que el habla tiene, entre otro, dos componentes que están doblemente disociados: la comprensión del habla y la emisión del habla.

Es así que usualmente se comienza planteando como hipótesis una independencia funcional, a partir de la aplicación del método de doble disociación de funciones cognitivas. Posteriormente se busca contrastarlo con algún método de neuroimagen. Lo más importante es que la disociación doble busca probar la existencia de procesos cognitivos separados o funcionalmente independientes. ${ }^{1}$

La disociación doble es un método muy útil para estudiar o descartar la existencia de interacciones entre tareas cognitivas, pero no está libre de complicaciones. Cabe preguntarnos si es efectivamente la aplicación concreta del método de doble disociación en un contexto específico nos está proporcionando evidencia suficiente de que hay independencia funcional entre las tareas cognitivas que estamos estudiando. Como veremos en la sección (iv), la respuesta a esta pregunta puede ser negativa y ello pone en duda el que las tareas cognitivas en cuestión sean realmente modulares.

Por el momento, abogamos a favor del uso de la doble disociación porque permite postular que hay una independencia, por lo menos mínima, entre dos sistemas, uno que lleva a cabo la tarea $A$ y otro que lleva a cabo la tarea $B$. El caso que objetaríamos es aquel en el cual las tareas cognitivas en cuestión no fueran independientes, compartieran recursos, en cuyo caso ambas tareas estarían averiadas al ocurrir una lesión cerebral, aparentando una falsa doble disociación.

\section{I.2.3. LA EVIDENCIA DE ACTIVIDAD CEREBRAL ESPECÍFICA O GRANULARIDAD GRUESA}

Persiste hasta nuestros días, por buenas razones, un rechazo a hablar de módulos entendidos como correlatos cognitivos de un sustrato físico alojado en el cerebro (esto es, como si efectivamente las capacidades cognitivas

1 Es posible que el método de doble disociación tenga inspiración en el siglo XIX y se haya fundamentado en recomendaciones metodológicas, como las ofrecidas por J. S. Mill (1882) acerca de qué es una buena explicación científica. Según este filósofo, una buena explicación del tipo « $A$ es causado por $X$ » debe, por un lado, mostrar que $A$ y $B$ son producidos por $X, Y$ y $Z$, pero $A$ y $C$ son producidos por $X, W$ y $Z$; y, por otro lado, que $A$ y $B$ son producidos por $X, Y$ y $Z$, pero únicamente $B$ es producido por $Y$ y $Z$. 
estuvieran «localizadas» y fueran reducibles a una región del cerebro). Hay al menos tres fuertes razones que contravienen a este «localizacionismo»: la flexibilidad adaptativa de la cognición (esto es, que si el cerebro contase con mecanismos heurísticos de procesamiento de información innatos, no podría explicarse lo bien que los humanos aprenden a resolver nuevos retos medioambientales y que implican un reclutamiento o reutilización neuronal); la eficiencia computacional de la cognición (que si el cerebro contase con mecanismos heurísticos de procesamiento de información innatos, ello implicaría una alta demanda de almacenamiento de mecanismos, lo cual lo haría computacionalmente ineficiente), y; la imposibilidad fáctica del localizacionismo (entendida como la ubicación topográfica reduccionista de capacidades cognitivas en el cerebro, la cual ha sido refutada). ${ }^{2}$

Ahora bien, el que las capacidades cognitivas no se localicen en un lugar específico del cerebro, es innegable que algo ocurre en él, y que sin el cerebro no podríamos llevar a cabo ningún tipo de procesamiento. La sutileza radica en explicar cómo y en qué medida se da esa activación cerebral. De modo que la modularidad involucra una relación de asociación entre tarea cognitiva y actividad cerebral. Sin embargo, esta relación no es reduccionista, pues el desempeño de una tarea cognitiva implica activación neuronal entre muchos otros elementos.

Ahora bien, para explicar cómo la ejecución de algunas tareas cognitivas se asocia a la activación de ciertas estructuras cerebrales particulares, es necesario distinguir tres tipos de activación: a) una correlación fuerte, $b$ ) una granularidad cerebro cognitiva gruesa y c) una granularidad cerebro cognitiva moderada. La activación del tipo a) diría que en el cerebro tenemos zonas que se correlacionan exclusivamente con el procesamiento de una y sólo esa tarea cognitiva particular (lo que equivaldría al localizacionismo clásico). Sin embargo, no parece haber evidencia empírica fuerte que apoye este tipo de activación. Por su parte, la activación del tipo b) dirá que cierta región del cerebro puede estar activa durante otros procesos cognitivos -lo cual implica que puede haber un traslape funcional-, pero que durante la asociación entre la tarea cognitiva y la actividad cerebral particulares, esta activación es de gran intensidad e involucra regiones bien definidas. Por último, la activación c) es aquella en la que la conjunción entre la activación asociada a la ejecución de la tarea cognitiva y la actividad cerebral es de poca intensidad e involucra regiones poco definidas (Hernández-Chávez 2016: 21).

2 Estas complicaciones se atenúan si postulamos que el correlato cerebral de activación que se asocia a un proceso cognitivo particular no se encuentra codificado genéticamente (no es innato), sino que se adquiere como resultado de un proceso de especialización o instrucción ambiental, tal como veremos en la siguiente sección. 
Así, tenemos que para poder hablar de modularidad se debe presentar activación del tipo $b$ ). La activación del tipo $a$ ) no parece ser empíricamente plausible, mientras que la activación del tipo $c$ ) es demasiado débil para poder inferir la presencia de modularidad.

Por tanto, una característica importante a favor de la existencia de un módulo cognitivo es el hecho de que se pueda determinar que existe un patrón de activación cerebral, asociado a la ejecución de la tarea cognitiva, en donde la activación sea de gran intensidad e involucre regiones cerebrales bien definidas, lo que llamamos granularidad cerebro cognitiva gruesa.

\section{I.2.4. ESPECIALIZACIÓN CEREBRO-COGNITIVA}

Cuando se busca una caracterización empírica de la modularidad, es de gran utilidad tomar en cuenta los procesos de especialización cerebral. Muy posiblemente, el aspecto más rico y enigmático de la modularidad es el cómo se dan los procesos de especialización anatómico-cognitiva. Por ejemplo, hablar un idioma es resultado de un proceso complejo que implicó recibir insumos específicos y sistemáticos que como consecuencia llevó a una especialización neuronal particular.

En tiempos recientes, se ha demostrado cómo la experiencia conlleva a cambios en la organización neural. Uno de los casos más claros es el ofrecido por Ptito \& Desgent (2006). Estos investigadores establecieron que el desarrollo cerebral es resultado de la interacción entre los componentes básicos del sistema nervioso y el medio ambiente, y que tal interacción puede modificar no solamente la organización de los sistemas neurales sino su función. En su estudio, Ptito \& Desgent (2006) reportan procesos de especialización cerebral de los sistemas visuales, tanto en animales como en humanos. Los autores dan a conocer hallazgos que muestran cómo ciertas modificaciones en la corteza que ayuda a distinguir estímulos visuales -como la pérdida de receptores sensoriales, traumatismos en los sistemas centrales del cerebro, etc.- producen cambios profundos en la estructura y función del sistema mismo. De modo que hay una reorganización de la corteza sensoriales visual en particular. ${ }^{3}$

3 Diversos estudios han sugerido que el tamaño del hipocampo posterior se relaciona con el grado de memoria, tal como reporta Maguire et al. (2000) sobre los choferes de taxi. En un caso similar, Elbert et al. (1995) reportaron que la representación cortical de los dedos de la mano izquierda ocupa un espacio mayor en los ejecutantes diestros de instrumentos de cuerda, que en los sujetos de control. Tal reorganización también se relaciona con la edad en que comenzaron a adquirir tal habilidad. 
El estudio del sistema visual es notorio en tanto que es parte constitutiva de diversos procesos cognitivos humanos, por ejemplo, para el reconocimiento de objetos, para distinguir colores, formas, personas, etc.

Otro estudio que ilustra el surgimiento de cambios cuantitativos y cualitativos en la organización neural, es el de Polk \& Hamilton (2006). Ellos revisan evidencia de especialización neuronal en dominios como la lectura, la escritura y la aritmética. Lo primero que debe notarse es que estas son capacidades bastante recientes en términos evolutivos, por lo cual difícilmente podrían ser producto de la selección natural. Adicionalmente, no se dan de manera automática, sino que requieren una instrucción específica y sistemática. Esta instrucción tiene como resultado la modificación de estructuras anatómicas concretas, en forma de cambios significativos y cualitativos en la organización cerebral en respuesta a las demandas culturales concretas. Notoriamente, los autores presentan resultados según los cuales la experiencia e instrucción de la lectura, la escritura y la aritmética conducen al desarrollo de nuevas áreas funcionales que se correlacionan con tales habilidades. Polk \& Hamilton (2006) explican cómo cada nueva habilidad está conformada por mecanismos más primitivos y básicos. Por ejemplo, la lectura implica procesos visuales básicos, el reconocimiento de distintas figuras, etc.; la escritura implica un control motor fino; y las matemáticas implican la habilidad de representar cantidades análogas. Esto les permitió sugerir que se podrían explicar o encontrar disfunciones como la dislexia y otros desórdenes en la lectura, la escritura y la aritmética, con el simple análisis de los subprocesos que componen a cada uno de ellos.

Por tanto, cuando se estudia un módulo particular es de crucial importancia hacer un estudio minucioso de los subprocesos básicos y capacidades cognitivas que lo componen. Asimismo, es necesario estudiar cómo tales capacidades se desarrollaron y fijaron en los individuos particulares dadas sus demandas ambientales hasta establecer una organización neuronal concreta.

En concreto, si buscamos una formulación de la modularidad que sea útil tanto para los estudios cognitivos como para la neurociencia social, es fundamental contar con una noción de módulo constituida a partir de los cuatro elementos: (1) la presencia de patrones típicos de disfunción, (2) la presencia de una disociación doble, (3) evidencia de actividad cerebral específica en términos de un patrón de activación no estrictamente localizacionista pero sí de granularidad gruesa, y, (4) un proceso de especialización cerebro-cognitiva.

Una vez expuesta esta noción cognitivo-neuronal de módulo, haremos uso de la misma para examinar uno de los módulos dedicados a la cognición social. Sin embargo, antes de ello, en el siguiente apartado exponemos una propuesta modular de la cognición social, aquella que pretende dar cuenta de la detección de trampa. 


\section{LOS MÓDULOS DE COGNICIÓN SOCIAL Y EL CASO DE LA DETECCIÓN DE} TRAMPA

La versión mas difundida de que los humanos poseemos un módulo de detección de tramposos, es la que defendieron los psicólogos evolucionistas. Apoyándose en estudios de neuroimagen, sugirieron que existen patrones particulares de activación cerebral cuando los sujetos detectan la violación de una regla social (Ermer et al. 2006).

Contextualizando un poco, los psicólogos evolucionistas afirmaban que dentro de la mente masivamente modular existe «un sistema neurocognitivo que está funcionalmente especializado para razonar sobre intercambios sociales» (Cosmides et. al. 2005, p. 505). La idea de estos psicólogos es que los seres humanos contamos con una facultad social que está formada por un conjunto de módulos que se interrelacionan. Cada uno de estos módulos está dedicado a procesar información sobre los sujetos de nuestra misma especie y las relaciones entre éstos. Dentro de estos módulos existe uno para detectar tramposos. ${ }^{4}$ Se consideran como tramposos a aquellos sujetos que buscan recibir los beneficios de los diferentes contratos sociales sin estar dispuestos a retribuir los costos que éstos conllevan. ${ }^{5}$ Originalmente, la postulación de este módulo se debió a consideraciones de tipo evolutivo y al apoyo proveniente de pruebas psicológicas. Veamos.

Respecto a las consideraciones evolutivas, Cosmides \& Tooby (1992) hacen uso de la teoría del «altruismo recíproco» de Robert Trivers como la única manera de explicar, desde el marco de la teoría de la selección natural, las conductas altruistas en los seres humanos y otros animales. La idea básica del altruismo recíproco es que los sujetos de una especie ayudan a sus congéneres siempre y cuando reciban también ayuda a cambio. Como ilustración de lo anterior, pensemos en un cazador recolector que ha tenido suerte al conseguir alimento, éste se comporta de manera altruista con otros cazadores porque espera una retribución a cambio, por ejemplo, que en ocasiones futuras se le compartan comida. La idea de Cosmides \& Tooby es que para que el altruismo recíproco sea posible los sujetos deben ser capaces de detectar tramposos, ya que éstos intentan recibir ayuda sin ofrecerla posteriormente. De este modo, tales psicólogos evolucionistas infieren que, dado que el altruismo recíproco es

4 Otro de los renombrados módulos sociales es el módulo de teoría de la mente, lo cual consiste en atribuir estados mentales a otros sujetos (Baron-Cohen et al. 1985). Para críticas a esta propuesta véase Gerrans \& Stone (2008).

5 Un contrato social se entiende en un sentido muy amplio, como un acuerdo tácito o explícito en el que un sujeto lleva a cabo una labor o actividad y espera ser beneficiado posteriormente. Por ejemplo, un contrato social es el acuerdo entre dos vecinos: $S$ cuida al perro de $T$ mientras $T$ está de viaje, por su parte $T$ riega las plantas de $S$ cuando éste no puede hacerlo. 
un hecho, en nuestra mente debe existir un módulo para detectar tramposos. ${ }^{6}$

El otro apoyo a favor del módulo de detección de tramposo de Cosmides \& Tooby proviene de pruebas de razonamiento, en particular, de la tarea de selección de tarjetas. ${ }^{7}$ Los sujetos no suelen responder de manera correcta a las versiones abstractas de la tarea de selección de tarjetas (Wason, 1966). Sin embargo, cuando una tarea de selección de tarjetas es reformulada en el marco de un contexto social, donde los sujetos tienen que descubrir quién está haciendo trampa, los sujetos ofrecen respuestas sistemáticamente correctas. Cosmides \& Tooby (1992) y otros psicólogos (Gigerenzer \& Hug, 1992) han relacionado la ejecución correcta de la tarea de selección de tarjetas con la detección de tramposos, y han defendido que éstas son evidencia a favor de la existencia de un módulo para detectarlos. La idea de estos psicólogos es que, en las versiones abstractas de la tarea de selección de tarjetas, los sujetos se equivocan porque éstas no se activa el módulo de detección de tramposos.

Como hemos repetido, las consideraciones evolucionistas y las pruebas de razonamiento son utilizadas por los psicólogos evolucionistas para apoyar la existencia de un módulo para detectar tramposos. Sin embargo, estos apoyos han sido duramente criticados (Buller, 2005). Como enmienda, los psicólogos evolucionistas ofrecieron evidencia neurológica para apoyar la existencia del módulo de detección de tramposos. En un número especial de Social Neuroscience, dedicado a la teoría de la mente, los psicólogos evolucionistas (Ermer, et al. 2006) sostienen que el módulo de detección de tramposos es un módulo porque: 1) existen disociaciones funcionales desencadenadas por el contenido que revelan un diseño para resolver tal problema adaptativo; 2) hay presencia de disociaciones neuronales ligadas a un daño cerebral o a

6 El argumento evolucionista de Cosmides y Tooby (1992) puede formularse como sigue:

- Existe altruismo en el ser humano y otras especies, lo cual es difícil de explicar dada la teoría de la selección natural.

- La única manera de explicar el altruismo (haciendo uso de la teoría de selección natural) es a través del altruismo recíproco.

- El altruismo recíproco es posible sólo si los sujetos cuentan con herramientas para detectar tramposos.

Por lo tanto,

La mente humana cuenta con un módulo para detectar tramposos.

7 En la primera versión de la tarea de selección de tarjetas (Wason 1966), que se conoce como la versión abstracta de esta prueba, se le presentan a los sujetos cuatro tarjetas y se les indica que éstas tienen de cada lado una letra y un número. Como los sujetos miran un lado de la carta, su trabajo consiste en voltear las tarjetas que muestren la verdad o falsedad de la siguiente oración: «si la tarjeta tiene una vocal en uno de sus lados, entonces tiene un número par en el otro lado». Se descubrió que comúnmente los seres humanos no respondemos correctamente esta tarea. Sin embargo, a partir de la versión abstracta han surgido diferentes versiones de tareas de selección obteniendo distintos resultados en cada versión (Evans \& Over, 1996). 
desórdenes de desarrollo que se observan con neuroimágenes; 3) presentan un desarrollo precoz robusto, y; 4) exhiben una uniformidad intercultural. Es importante señalar que anteriormente los psicólogos evolucionistas no se comprometían de manera explícita con la idea de que un módulo estuviera vinculado a «disociaciones neuronales».

El experimento que plantearon los psicólogos evolucionistas para evidenciar la existencia de disociaciones y de ahí la presencia de un módulo para detectar tramposos consistió en pedirle a doce sujetos que resolvieran seis problemas de selección de tarjetas: dos en contratos sociales, dos en reglas de precaución y dos en reglas que describieran preferencias o hábitos (Ermer et al., 2006). En el caso de la tarea de selección de tarjetas, se buscaba averiguar quién había violado un contrato social, para así determinar si se cumplía la regla: «si el sujeto toma alcohol, entonces debe ser mayor de edad». Un caso de regla de precaución durante las tareas de selección enfocadas en situaciones de riesgos era «si se trabaja con pacientes con tuberculosis, entonces se debe usar un tapaboca». Por último, se presentaba una tarea de selección de tarjetas en la que se describían hábitos y consistía en determinar si una regla indicativa se cumplía. Por ejemplo, «si el sujeto es estudiante de biología, entonces tiene gusto por salir de día de campo». Un punto importante del experimento es que los psicólogos evolucionistas dividieron en dos partes la tarea de selección de tarjetas: una fase interpretativa, en donde el sujeto examinaba las opciones de la tarea de selección de tarjetas, y una segunda fase, en donde el sujeto determinaba la selección de la(s) tarjeta(s).

En cuanto a los resultados sobre qué tan bien ejecutaban la tarea de selección de tarjetas, los psicólogos evolucionistas mostraron que durante los contratos sociales y en las reglas de precaución los sujetos tenían una ejecución similar ( $81 \%$ y $83 \%$ de respuestas correctas respectivamente), mientras que durante las reglas descriptivas tuvieron una ejecución significativamente más baja (59\% de respuestas correctas). Este resultado parece indicar, según los psicólogos evolucionistas, que el mecanismo que procesa inferencias donde intervienen contratos sociales y reglas de precaución es distinto de aquel a cuando se tenían que procesar reglas descriptivas. ¿Lo anterior implicaría que existe un mismo módulo para detectar tramposos que para detectar si se viola una regla de precaución? Los psicólogos evolucionistas recurrieron a la imagenología por fMRI para mostrar que efectivamente se daba un tipo de activación cerebral diferenciada cuando ejecutaban tareas de selección de tarjetas donde se violaba un contrato social, que cuando se violaba una regla de precaución.

Los resultados obtenidos son los siguientes. En cuanto a las imágenes cerebrales que obtuvieron señalaron que, en la fase interpretativa, durante las tareas de contrato social se producía una mayor activación en las regiones 
temporo-parietales; mientras que durante la toma de decisiones se producía una mayor activación en la corteza prefrontal dorsolateral. Durante la fase interpretativa las tareas de contrato social, con respecto a las tareas de precaución, se activaban la corteza anterior temporal derecha (Área de Brodmann (AB) 20) y la corteza posterior temporal izquierda (AB 21), junto con la corteza cingulada posterior (AB 23). En cuanto a la segunda fase, es decir, durante la toma de decisiones, se encontró que la tarea de precaución, comparada con la del contrato social, mostró activaciones en la corteza prefrontal media y la ventral (AB 6, 9, 46 y 47), la corteza temporal media y posterior ( $\mathrm{AB} 21$ y 41), la región superior occipital ( $\mathrm{AB} 18$ y 37), así como en la ínsula derecha (AB 13) y la circunvolución del cíngulo (AB 24) (Ermer et al. 2006, p. 207). De los resultados anteriores, Ermer et al. (2006) sostienen que durante las tareas de selección de contratos sociales se activan distintas áreas a cuando se ejecutan tareas de precaución. Así respaldan su afirmación de que las reglas de contratos sociales y las reglas de precaución activan distintos módulos (Ermer et al. (2006, p. 209). Los autores explícitamente afirman que si las reglas de contratos sociales y las reglas de precaución fueran iguales, entonces activarían las mismas áreas del cerebro, esto es, cuando el sujeto se enfrentara a un contrato social y cuando el sujeto se enfrentara a una regla de precaución. Esto, sin embargo, consideran que no sucede.

En suma, las consideraciones evolucionistas y los experimentos psicológicos, como la tarea de selección de tarjetas, fueron recurrentemente utilizados por los psicólogos evolucionistas para apoyar la existencia de un módulo para detectar tramposos. A estos apoyos se han sumado otros resultados que parecen mostrar que se activan diferentes áreas del cerebro cuando los sujetos interpretan reglas de contratos sociales y cuando interpretan reglas de precaución. Dado lo anterior, ¿podríamos considerar que hay buenas razones para sostener la existencia de un módulo de detección de tramposos?

\section{MODULARIZANDO Y DESMODULARIZANDO LA COGNICIÓN SOCIAL}

En esta sección intentaremos mostrar por qué el módulo de detección de tramposos no encuentra apoyo sólido a la luz de la evidencia neurológica. Para ello nos centraremos en dos de las características de la modularidad: la doble disociación y la especialización cerebral. Nótese que la primera es una preocupación que también ocupa a Ermer et al. (2006).

La correcta identificación de un patrón específico de activación cerebral dependerá de si las disociaciones propuestas pueden satisfacerse. Por cuestiones de espacio, solamente mencionaremos algunas observaciones respecto a la presencia de un patrón de activación cerebral con una granularidad gruesa. Sería necesario que la activación cerebral asociada a la ejecución de la tarea cognitiva sea de gran intensidad e involucre a regiones cerebrales bien 
definidas. A este respecto sólo anotamos que se necesitan estudios adicionales para distinguir cuáles son las áreas cerebrales que diferencialmente se activan cuando un sujeto detecta que alguien viola un contrato social, en contraste con la activación cerebral diferencial en tareas relacionadas. Sin embargo, como veremos enseguida, las disociaciones que plantean sus defensores son problemáticas.

\section{III.1. EL MÓDULO DE RAZONAMIENTO SOCIAL Y LA DISOCIACIÓN DOBLE}

Como hemos expuesto en el apartado anterior, los psicólogos evolucionistas consideran que los módulos deben contar con las siguientes características: (1) disociaciones funcionales desencadenadas por un contenido que revelen la presencia de un diseño adaptativo, (2) disociaciones neuronales asociadas a daño cerebral y desórdenes del desarrollo, (3) un desarrollo precoz robusto, y, (4) una uniformidad intercultural. Como vimos, los psicólogos evolucionistas se concentran en la segunda característica y no desarrollan la tercera y cuarta característica.

Un problema con las disociaciones que Ermer et al. (2006) plantean es que no se está sometiendo a prueba si el razonar sobre la violación de reglas en contratos sociales es independiente del razonar sobre la violación de reglas de precaución. Es decir, no están considerando seriamente la posibilidad de que ambas tareas sean parte de un mecanismo general de razonamiento sobre distintos dominios. Lo que están haciendo es postular que efectivamente se trata de dos tareas independientes, para subsecuentemente intentar confirmarlas con escaneos cerebrales que asumen de antemano una independencia funcional entre ambas. Esta dificultad es inherente al uso de las disociaciones dobles. El riesgo es que la aparente independencia funcional de las dos tareas cognitivas puede en realidad esconder las siguientes dos situaciones:

De acuerdo con los datos conductuales, en el grupo de sujetos $S 1$, la tarea cognitiva $A$, razonamiento sobre intercambios sociales (círculo verde), está deteriorada porque presenta un daño en el subproceso $x$ (círculo blanco a la izquierda), pero la tarea cognitiva $B$, razonamiento sobre normas de precaución (círculo rojo), está intacta. Estos datos conductuales, entonces, no necesariamente implican que $A$ y $B$ sean completamente independientes, pues 
puede suceder que haya un área de traslape entre ellas, como se muestra en el esquema de la Figura 1:

Figura 1:

A

B

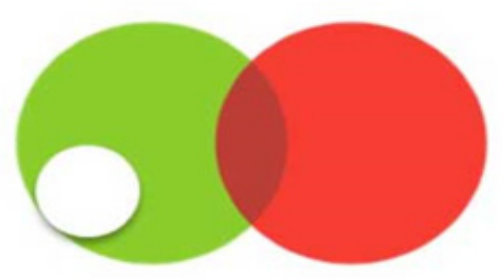

Recíprocamente, de acuerdo con los datos conductuales en el grupo de sujetos $S 2$, la tarea cognitiva $B$, razonamiento sobre normas de precaución (círculo rojo), podría estar deteriorada porque presenta un daño en el subproceso $y$ (círculo blanco a la derecha), pero la tarea cognitiva $A$, razonamiento sobre intercambios sociales (círculo verde), está intacta. Se tendría algo como el esquema de la Figura 2:

\section{Figura 2:}

A B

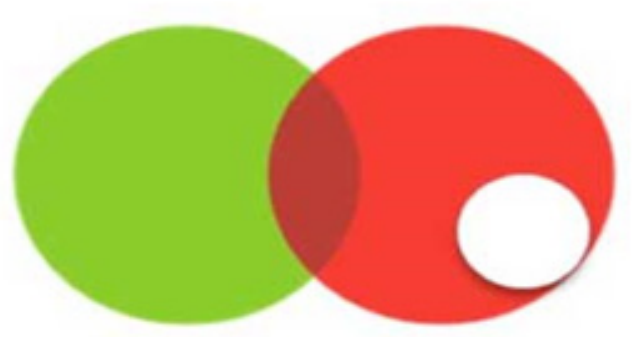


En otras palabras, el uso que hacen Ermer et al. (2006) del método de doble disociación busca confirmar que en un grupo de sujetos $S 1$, la tarea cognitiva $A$ está disociada de una tarea cognitiva $B$; y a la inversa, que en el grupo de sujetos $S 2$ la tarea $B$ está disociada de la tarea $A$. Sin embargo, esto es muy difícil de establecer.

En concreto, la evidencia que ofrecen Ermer et al. (2006) no excluye la posibilidad de que el módulo para detectar tramposos (a) sea en realidad parte del módulo de razonamiento sobre problemas de dominios de contenido más generales (b). Es muy posible que lo que en realidad estén reportando estos datos conductuales sea una independencia funcional mínima, o más bien nula, de $A$ respecto a $B$.

Adicionalmente, las disociaciones que plantean Ermer et al. (2006) no son de tareas cognitivas equivalentes, algunas incluyen necesariamente la atribución de estados mentales, mientras que en las reglas de precaución o en las reglas descriptivas no necesariamente se atribuyen estados mentales. Por ejemplo, una tarea de selección de tarjetas puede contener la regla descriptiva: «si comes carne roja, entonces tomas vino tinto». En dicha regla no se requiere atribuir estados mentales. Por el contrario, para resolver tal prueba psicológica se seguiría simplemente el uso de la lógica (en particular el uso de las tablas de verdad (Wason, 1966)). Probablemente, la diferencia de actividad neuronal que aparece durante las tareas de selección de tarjetas es producto de la necesidad de atribuir estados mentales, pues se busca identificar quién viola un contrato social, lo cual no es necesario durante las tareas de selección ante reglas descriptivas o de precaución.

Por tanto, consideramos que la evidencia de neuroimágenes cerebrales y dobles disociaciones que utilizan Ermer et al. (2006) no son suficiente para saber si efectivamente el razonamiento sobre intercambios sociales es independiente del razonamiento sobre normas de precaución. Así, siguiendo nuestra noción cognitiva-neuronal de módulo y la preocupación misma de Ermer el al. (2006), el supuesto módulo de detección de tramposos no satisface la especificación de que un módulo debe estar apoyado en disociaciones genuinas.

\section{III.2. MÓDULO DE RAZONAMIENTO SOCIAL Y ESPECIALIZACIÓN CEREBRAL}

En la sección anterior pusimos en duda la validez de las disociaciones, a saber, el tipo de evidencia (1) y (2), y el tipo de evidencia (3) que se deriva de la suposición de que existen disociaciones -nótese que no se está poniendo a prueba si las disociaciones efectivamente existen-. En esta sección nos centraremos en debatir el tipo de evidencia (4), la más fuerte de todas, pues negar la existencia de un módulo de razonamiento de contratos sociales, $y$, 
específicamente para detectar tramposos, pone en duda no sólo a este módulo particular sino la existencia de módulos sociales en general.

Como revisamos, psicólogos evolucionistas como Ermer et al. (2006) consideran que la mente humana posee, en mayor medida, capacidades mentales funcionalmente especializadas en la solución de un problema adaptativo recurrente, que son además específicas de dominio. Ejemplos son los procesos algorítmicos, las capacidades inferenciales, las reglas heurísticas, etc. Tales dispositivos con contenido específico no podrían explicarse como derivados de la lógica y la matemática, sino «que se desarrollan en la mente humana sin instrucción» (Ermer et al. 2005, p. 197). ${ }^{8}$

La idea de que pueda haber mecanismos cognitivos sociales que se desarrollen sin instrucción es arriesgada. Por ello queremos contrastarla con el trabajo de Polk \& Hamilton (2006) mencionados en el punto (4) de la noción cognitivo-neuronal de módulo. Ellos explican cómo los dominios particulares de la lectura, la escritura y la aritmética están conformados por mecanismos más primitivos y básicos. Por ejemplo, la lectura implica procesos visuales básicos, el reconocimiento de distintas figuras, etc.; la escritura implica un control motor fino; y las matemáticas implican la habilidad de representar cantidades análogas. Esto les lleva a sugerir que se podrían explicar o encontrar disfunciones como la dislexia y otros desórdenes en la lectura, la escritura y la aritmética, analizando los subprocesos que componen a cada uno de ellos.

De manera similar, cabe presumir que el razonamiento sobre normas de precaución, el razonamiento sobre normas de contrato social y módulos similares, también están constituidos por mecanismos más básicos como el reconocimiento de objetos animados, objetos inanimados, rostros, subprocesos de memoria de largo plazo, de corto plazo, procesos de asociación mínimos, hasta ascender a unos más complejos, entre otros mecanismos. Esto nos permite suponer que la composición de neuroimágenes cuando se llevan a cabo el razonamiento sobre normas de intercambio social seguramente implican a un conjunto de subprocesos como los anteriores. Asimismo, surge la posibilidad de que un déficit selectivo que erróneamente se esté tomando como evidencia a favor de un módulo cognitivo funcionalmente independiente y único, más bien sea parte constitutiva de un proceso extendido.

\section{III.3. SOBRE LA UNIFORMIDAD CULTURAL}

Si bien Ermer et al. (2006) aducen como evidencia confirmatoria el que los módulos posean una uniformidad intercultural -su evidencia tipo (4)-,

8 Según Ermer et al. (2006), estas capacidades contrastan con otros sistemas inferenciales que también poseemos en menor medida, a saber, sistemas inferenciales sin contenido específico y de dominio general. 
en su escrito no ofrecen experimentos que muestre que en todas las culturas humanas, o en la mayoría de ellas, los sujetos desarrollemos módulos de razonamiento social con base en la misma pauta de desarrollo. Esto es, si bien puede haber intuiciones que hagan plausible suponerlo, no reportan estudios extensos de que uniformemente los humanos, independientemente de la cultura a la que pertenezcamos, desarrollemos módulos que nos permitan detectar infractores en nuestra comunidad. Por lo anterior, no podemos validar el punto (4) de la propuesta de Ermer et al. (2006): la existencia de una uniformidad intercultural como evidencia a favor de que poseemos un módulo para detectar tramposos. Esto a su vez pone en entredicho su punto (3), a saber, que los mismos exhiban desarrollo precoz robusto.

La búsqueda de módulos para capacidades como el razonamiento social coincide con el anhelo de encontrar rasgos humanos universales. Por ejemplo, si eres capaz de atribuirle a un sujeto de cualquier cultura una teoría de la mente, o un razonamiento social, entonces presumiblemente se trate de un rasgo específicamente humano y universalizable (un tipo de recapitulacionismo antropológico que explica cómo el sujeto actual tiene la capacidad de entender a sujetos evolutivamente anteriores). Si esto fuera así, podríamos plantear un experimento mental en donde, si tuviéramos una máquina del tiempo con la cual viajar hacia atrás, podríamos platicar con Platón y entender su contexto. Esto, sin embargo, es negado por la diversidad cultural. La crítica más fuerte a la búsqueda de universales biológicos, proviene de considerar que la mente que actualmente tenemos, es resultado tanto de una serie de presiones culturales, como del conjunto de interacciones que adquirimos desde la infancia.

Tenemos, pues, dos visiones distintas respecto a la mente y la cognición, una de ellas apela al pasado evolutivo y supone que las capacidades en cuestión tuvieron una ventaja adaptativa, nos ayudaron a vivir y por ello se transmitieron. En contraste con una visión desarrollista o antropológica que incorpora el entorno bio-psico-social como fuerzas que promovieron el establecimiento de la capacidad en cuestión, como un módulo para la cognición social.

\section{CONCLUSIONES}

Hoy en día es bien aceptada la idea de que tenemos mecanismos cognitivos especializados en dominios específicos. A esta idea responde la modularidad cognitiva. Ha habido distintas maneras de caracterizar lo que es un módulo, los rasgos más usuales que lo definían eran: especificidad de dominio, encapsulamiento, independencia funcional y una historia adaptativa o filogenética particular. En este trabajo comenzamos haciendo un recuento del debate acerca de la arquitectura de la mente y presentamos una noción de módulo que incluye elementos como: la presencia de patrones típicos de disfunción, la disociación doble, la evidencia de actividad cerebral específica en 
términos de una granularidad gruesa, y, un proceso de especialización cerebrocognitiva. En segundo lugar, expusimos una de las propuestas que busca dar cuenta del razonamiento sobre aspectos sociales, i.e., la defendida por los psicólogos evolucionistas, en particular, el supuesto módulo diseñado por la selección natural para detectar tramposos. En la última parte, contrapusimos la noción de módulo cognitivo-neuronal con el módulo de razonamiento social, y argumentamos por qué no es posible defender la existencia del módulo.

La postulación de módulos particulares ha proliferado en la literatura neuropsicológica y neurocientífica en general. En este trabajo decidimos evaluar un módulo de razonamiento social, i.e., uno postulado para detectar tramposos. La evidencia más reciente que aducen los psicólogos evolucionistas a favor de este módulo es la proporcionada por las «disociaciones neuronales», por ello nos concentramos en analizar cómo aplican tales disociaciones. Quienes aceptan un módulo como el anterior sostienen que nuestra estructura cerebral da lugar a una mente modular que media nuestra cognición social, esto es, que hay módulos que subyacen a nuestras interacciones y relaciones sociales. Como vimos, Ermer et al. (2006) plantean una disociación entre: un módulo de detección de tramposos y otros mecanismos, como aquel que se aplica a las normas de precaución. Sin embargo, dichos autores no están poniendo a prueba si estos dos tipos de razonamiento son independientes; únicamente la están dando por sentada y proponiendo evidencia de escaneo cerebral a favor de la misma. Adicionalmente, las disociaciones que se plantean en Ermer et al. (2006) no son de tareas cognitivas equivalentes, unas incluyen la atribución de estados mentales, mientras que otras no las incluyen. Por tanto, se concluyó que la evidencia de neuroimágenes cerebrales y dobles disociaciones que utilizan los psicólogos evolucionistas no es suficiente para saber si efectivamente el razonamiento sobre intercambios sociales es independiente del razonamiento sobre normas de precaución, y ello invalida la supuesta modularidad de ese proceso cognitivo.

Una segunda crítica que hicimos a Ermer et al. (2006) es a su evidencia (4) sobre la homogeneidad cultural del módulo de cognición social. Objetamos que puede presumirse que el razonamiento sobre normas de precaución, el razonamiento sobre normas de intercambio social y módulos similares, también están constituidos por mecanismos más básicos como el reconocimiento de objetos animados, objetos inanimados, rostros, subprocesos de memoria de largo plazo, de corto plazo, entre muchos otros. Esto nos permite suponer que la composición de neuroimágenes cuando se lleva a cabo una tarea en que se debe descubrir quién viola un contrato social, seguramente implica a todos los subprocesos anteriores. Directamente relacionado con esto, no hay evidencia sólida que muestre que en la mayoría de las culturas humanas los sujetos desarrollemos módulos de razonamiento social con la misma pauta y 
los mismos resultados.

Después de señalar las debilidades del supuesto módulo para detectar tramposos surge la pregunta de si otros mecanismos de cognición social, como lo es la teoría de la mente, pueden considerarse como modulares. Proponemos que es necesario hacer estudios cuidadosos de cada uno de los supuestos módulos de cognición social. Para ello sería necesaria la revisión del supuesto módulo para atribuir estados mentales, que nuevamente incluiría la evaluación de los aspectos teóricos y empíricos sobre el tema (Adams, 2011). Creemos que no hay indicios sólidos para postular que mecanismos como la atribución de estados mentales puedan ser modulares porque, al parecer tampoco constituyen disociaciones dobles (Gerrans y Stone, 2008). Sin embargo, se requieren estudios adicionales al respecto. Otro punto que también nos parece importante desarrollar en el campo de la cognición social es aquel sobre el razonamiento deóntico, según el cual nuestra arquitectura mental está estructurada por conceptos como la obligación, prohibición y permiso en el ámbito social (Cummins, 2000). Estos estudios forman parte de los futuros trabajos, los cuales suponen el trabajo multidisciplinario de la neurociencia social en el que se conjugan la psicología social, la psicología cognitiva, las neurociencias y, por supuesto, el análisis conceptual de la filosofía.

\section{REFERENCIAS BIBLIOGRÁFICAS}

ADAMS, M (2011). Modularity, theory of mind, and autism spectrum disorder. Philosophy of Science, 78, 763-773. doi:10.1086/662269.

BARON-COHEN, S., Frith, U., \& Leslie, A (1985). Does the autistic child have a 'theory of mind'?. Cognition, 21,37-46. doi:10.1016/0010-0277(85)900228.

BULLER, D (2005). Adapting minds: evolutionary psychology and the persistent quest for human nature. Cambridge, MA: MIT Press. https://mitpress.mit. edu/books/adapting-minds

CARRUTHERS, P (2006). The architecture of the mind: Massive modularity and the flexibility of thought. Oxford, England: Clarendon Press. https:// books.google.com.mx/books/about/The Architecture of the Mind.

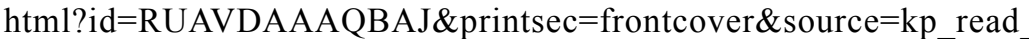
button\&redir_esc $=\mathrm{y} \# \mathrm{v}=$ onepage $\& \mathrm{q} \& \mathrm{f}=$ false

COSMIDES, L., \& TOŌBY, L. (1992). Cognitive adaptations for social exchange. En J. Barkow, L. Cosmides \& J. Tooby (Eds.), The adapted mind: evolutionary psychology and the generation of culture (pp. 163-228). New York, NY: Oxford University Press. https://psycnet.apa.org/record/1992-98504-003. 
COSMIDES, L., TOOBY, J., FIDDICK, L., \& BRYANT, G (2005). Detecting cheaters. Trends in cognitive science, 9, 505-506. DOI: https://doi. org/10.1016/j.tics.2005.09.005

CUMMINS, D. (2000). How the social environment shaped the evolution of mind. Synthese, 122, 3-28. https://doi.org/10.1023/A:1005263825428

DEHAENE, S., \& COHEN, L (1991). Two mental calculation systems: A case study of severe acalculia with preserved approximation. Neuropsychologia, 29, 1045-1074. doi:10.1016/0028-3932(91)90076-k

GERRANS, P., \& STONE, V (2008). Generous or parsimonious cognitive architecture? Cognitive neuroscience and theory of mind. British Journal of Philosophy of Science, 59, 121-141. https://doi.org/10.1093/bjps/axm038

GIGERENZER, G., \& HUG, K (1992). Domain-specific reasoning: Social contracts, cheating, and perspective change. Cognition, 43, 127-171. DOI: 10.1016/0010-0277(92)90060-u

ELBERT, T., PANTEV, C., WIENBRUCH, C., ROCKSTROH, B., \& TAUB, E (1995). Increased cortical representation of the fingers of the left hand in string players, Science, 270, 305-307. DOI: 10.1126/science.270.5234.305

ERMER, E., GUERIN, S., COSMIDES, L., TOOBY, J., \& MILLER, M (2006). Theory of mind broad and narrow: Reasoning about social exchange engages ToM areas, precautionary reasoning does not. Social Neuroscience, 1, 196-219. DOI: 10.1080/17470910600989771

EVANS, J., \& OVER, D (1996). Rationality and reasoning, East Sussex, England: Psychology Press. https://doi.org/10.1016/0010-0277(93)90039-X

FODOR, J.(1983). The modularity of mind: An essayon faculty psychology, Cambridge, MA: MIT Press. DOI: https://doi.org/10.1017/CBO9780511814273.046

HERNÁNDEZ-CHÁVEZ, Paola (2016), Modularidad cognitiva y especialización cerebral (2016), CEFPSVLT-SEP. Mexico. https://www.academia. edu/33396673/Modularidad_Cognitiva_y_Especialización_Cerebral

KARMILOFF-SMITH, A (2011). Static snapshots versus dynamic approaches to genes, brain, cognition, and behavior in neurodevelopmental disabilities. International review of research in developmental disabilities: early development in neurogenetic disorders, 40, 1-15. https://doi.org/10.1016/ B978-0-12-374478-4.00001-0

MACHERY, E (2007). Massive modularity and brain evolution. Philosophy of Science, 74, 825-838. https://doi.org/10.1086/525624

MAGUiRE, E. A., GADIAN, D. G., JOHNSRUDE, I. S., GOOD, C. D., ASHBURNER, J., FRACKOWIAK, R. S., \& FRITH, C. D. (2000). Navigation-related structural change in the hippocampi of taxi drivers. Proceedings of the National Academy of Sciences, 97(8), 4398-4403. https://doi.org/10.1073/pnas.070039597

MILL, J. S (1882). A system of Logic, New York, NY: Harper \& Brother Publishers. https://www.gutenberg.org/files/26495/26495-pdf.pdf

MITHEN, S (1996). The prehistory of mind: a search for the origins of art, religion, and science, London, England: Thames and Hudson. https://www.journals. uchicago.edu/doi/abs/10.1086/jar.52.4.3630315 
PARSONS, L., \& Osherson, D (2001). New evidence for distinct right and left brain systems for deductive versus probabilistic reasoning. Cerebral Cortex, 11, 954-965. https://doi.org/10.1093/cercor/11.10.954

PATTERSON, L., \& Kay, J (1982). Letter-by-letter reading: Psychological descriptions of a neurological syndrome, Quarterly Journal of Experimental Psychology, 34, 411-441. https://doi.org/10.1080/14640748208400852

POLK, T., \& Hamilton, J (2006). Reading, writing, and arithmetic in the brain: Neural specialization for acquired functions, En P. Baltes, P. Reuter-Lorenz \& F. Rosler (Eds.), Lifespan Development and Brain: The perspective of biocultural co-constructivism (pp. 183-199). New York, NY: Cambridge University Press. DOI: 10.1017/CBO9780511499722.010

PINKER, S (1994) The language instinct: how the mind creates language. New York, NY: HarperCollins Publishers. https://stevenpinker.com/publications/ language-instinct

PTITO, M., \& Desgent, S. (2006). Sensory input-based adaptation and brain architecture. En P. Baltes, P. Reuter-Lorenz \& F. Rosler (Eds.), Lifespan Development and Brain: The perspective of biocultural co-constructivism (pp. 111-133). New York, NY: Cambridge University Press. DOI: 10.1017/ CBO9780511499722.007

SPERBER, D. (1994). The modularity of thought and the epidemiology of representations. En L. Hirschfeld \& S. Gelman (Eds.), Mapping the mind: Domain specificity in cognition and culture (pp. 39-67). Ney York, NY: Cambridge University Press. DOI: https://doi.org/10.1017/ CBO9780511752902.003

SPERBER, D. (2002). In defense of massive modularity. En I. Dupoux (Ed.), Language, Brain, and Cognitive Development (pp. 47-57). Cambridge, MA: MIT Press. http://www.dan.sperber.fr/wp-content/uploads/2001_indefense-of-massive-modularity.pdf

Paola Hernández-Chávez es investigadora del ICS-UJED, donde también es profesora del Posgrado en Ciencias y Humanidades. Es Fellow at the Center for Philosophy of Science, University of Pittsburgh. Miembro del Sistema Nacional de Investigadores, Conacyt, Nivel I.

Líneas de investigación:

Metodología de la Neurociencia, Ciencias Cognitivas, Disfunciones Cognitivas, Filosofía de la Biología

Publicaciones recientes:

(2020). «Trampa y engaño. Algunas observaciones empíricas», en García Campos, J., Hernández Chávez, P., Sarabia López, S. Atilano Barbosa, D. Mente y Cultura, Revista del Instituto Nacional de Psiquiatría Ramón de la Fuente Muñiz 1(1). 
(2019). «Blinded by Biology: Bio-inspired Tech-Ontologies in Cognitive Brain Sciences», en Compagnoni A., Casey W., Cai Y., Mishra B. (eds), Bioinspired Information and Communication Technologies, vol 289. Springer, pp 58-69.

Correo electrónico: hcpaola@gmail.com

Jonathan García-CAmpos es profesor del ICS-UJED. Miembro del Sistema Nacional de Investigadores, Conacyt, Nivel I.

Líneas de investigación:

Epistemología contemporánea, Teorías del razonamiento y racionalidad, La intersección entre evolución y cognición, Historia y Filosofía de la Psicología

Publicaciones recientes:

2019. «¿Qué es un contexto para las teorías cognitivas del razonamiento?», Crítica. Revista Hispanoamericana de Filosofia. 51, 151, pp. 85-115

2019. «La Psicología Evolucionista y la idea de unidad en las ciencias», Entreciencias:

Diálogos en la Sociedad del Conocimiento, 7, 21, pp. 107-120.

Correo electrónico: jonatangarcia@ujed.mx 